\title{
Local Learning Collaboratives to Improve Quality for Chronic Kidney Disease (CKD): From Four Regional Practice-based Research Networks (PBRNs)
}

\author{
Paula Darby Lipman, PhD, and Cheryl B. Aspy, PhD
}

Background: Four practice-based research networks (PBRNs) participated in a project to increase the diffusion of evidence-based treatment guidelines for chronic kidney disease (CKD). A multicomponent organizational intervention engaged regionally proximal primary care practices in a series of facilitated meetings, referred to as local learning collaboratives (LLCs).

Methods: The 2-wave strategy began with 8 practices in each PBRN receiving practice facilitation and subsequently joining an LLC. A sequential mixed-methods design addressed the conduct, content, and fidelity of the intervention; clinicians in 2 PBRNs participated in interviews, and PBRN coordinators reflected on implementation challenges.

Results: LLCs were formed in 3 PBRNs, with 121 monthly meetings held across 20 LLCs. Slightly more than half of the participants were clinicians. Qualitative data suggest that clinicians increased the priority for CKD care, improved knowledge and skills, were satisfied with the project, and attempted to improve care. Implementation challenges were encountered and concerns about sustainability expressed.

Conclusion: While PBRNs can successfully leverage resources to diffuse treatment guidelines, and LLCs are well-accepted by clinical staff, the formation of LLCs was not feasible for 1 PBRN, and others struggled to meet regularly and have performance data available despite logistic support. ( $\mathrm{J}$ Am Board Fam Med 2016;29:543-552.)

Keywords: Chronic Renal Insufficiency, Cooperative Behavior, Learning, Practice-based Research, Primary Health Care

Between 1988 to 1994 and the 2003 to $2006 \mathrm{Na}$ tional Health and Nutrition Examination Surveys, the prevalence of chronic kidney disease (CKD) among individuals $\geq 60$ years old increased from $18.8 \%$ to $24.5 \% .^{1} \mathrm{CKD}$ is associated with a 5 -year all-cause mortality rate of $24 \%$ and a $20 \% 5$-year requirement for transplant or dialysis. ${ }^{2}$ The burden of CKD and accompanying morbidity and mortal-

This article was externally peer reviewed.

Submitted 29 January 2016; revised 12 April 2016; accepted 19 April 2016.

From Westat, Rockville, MD (PDL); and the Department of Family \& Preventive Medicine, University of Oklahoma Health Sciences Center, Oklahoma City (CBA).

Funding: This work was supported by a grant from the Agency for Healthcare Research and Quality (1R18HS019945).

Conflict of interest: none declared.

Corresponding author: Paula Darby Lipman, PhD, Westat, 1600 Research Blvd, Rockville, MD 20850 (E-mail: paulalipman@westat.com). ity is especially heavy for the poor, minorities, those with poor literacy, and safety-net patients. ${ }^{3}$

Guidelines have been available since $2002,{ }^{4}$ and there is evidence that guideline-based care can delay CKD progression and reduce mortality. ${ }^{5-8}$ The implementation of recommendations most applicable to primary care ${ }^{4}$ involves several care processes, ${ }^{9,10}$ including documenting diagnosis, ordering appropriate tests, discontinuing inappropriate medications, prescribing new medications, managing diabetes and cardiovascular disease risk factors, educating patients, administering appropriate immunizations, and referring patients with advanced disease. While there is some controversy regarding possible overdiagnoisis of CKD using estimated glomerular filtration rate (eGFR) as diagnostic criteria for stage $3 \mathrm{~A} \mathrm{CKD}$ without proteinuria testing (especially in the elderly, because of the age-related 
decline in eGFR), ${ }^{11,12}$ this target behavior seemed appropriate as the project was designed to improve implementation of current CKD guidelines, with which almost $50 \%$ of primary care physicians report being unfamiliar.

Dissemination, implementation, and diffusion of guideline recommendations remain suboptimal, ${ }^{13-15}$ leading to an increase in studies of approaches to improve guideline uptake in primary care. ${ }^{16}$ These implementation strategies are "methods or techniques used to enhance the adoption, implementation, and sustainability of a clinical program or practice." ${ }^{\prime 17}$ For example, although vast sums are spent on continuing medical education, ${ }^{18}$ evidence of effectiveness in translating research into practice has yet to emerge. ${ }^{19}$ Peer-to-peer learning has been found to motivate practice change, with formal learning collaboratives designed to create competition and urgency. ${ }^{20,21}$ Learning collaboratives typically involve large numbers of practices that receive education/ information, create quality improvement (QI) teams, perform periodic medical record reviews, develop registries, and strive to implement evidence-based strategies over time. ${ }^{22}$

This article describes a multicomponent organizational intervention that engaged small groups of regionally proximal practices in facilitated meetings focused on care of patients with or at risk of CKD. These "local" learning collaboratives (LLCs), defined as a "data-driven group/network of change teams from organizations with commonality of care that work collectively to enhance performance through process improvement," $23(\mathrm{p} 391)$ are a scaled down version of more common breakthrough learning collaboratives championed by the Institute for Healthcare Improvement and others. Our experience suggests the LLCs are well-accepted by clinicians and staff, ${ }^{24}$ and can achieve the same motivation, competition, and collaborative learning generated in larger collaboratives through more frequent, briefer meetings held in more convenient locations. Optimally, LLCs meet monthly (in person), with fewer than 10 individuals, and have the capacity to generate practice performance data for feedback and comparison, sufficient meeting support, and an agenda with clear objectives. Participants are expected to improve guideline implementation, share positive anecdotes and lessons learned, and create practical solutions for common implementation problems. Forming small groups of cli- nicians interested in practice improvement and sharing best practices can affect factors driving intention to change (ie, beliefs about capabilities and consequences) as well as improve knowledge and skills pertaining to the care of patients with CKD.

In 2010 a consortium of practice-based research networks (PBRNs) was funded by the Agency for Healthcare Research and Quality to improve implementation in primary care of the National Kidney Foundation Chronic Kidney Disease guidelines. The project was designed to evaluate how PBRN resources can be leveraged to demonstrate the effectiveness of a strategy to disseminate and diffuse guidelines of importance in primary care. We aimed to improve the implementation of key guidelines as evidence of the effectiveness of our strategy, which involved practice facilitation (wave I) followed by the formation of LLCs (wave II). Given evidence of effectiveness of this 2-wave strategy, ${ }^{25}$ a secondary study aim was to conduct a mixed-methods process evaluation to examine lessons learned, including (1) whether the LLC approach was implemented as planned, (2) the CKDrelated QI goals engaged in, and (3) the experiences of participating clinicians. Qualitative data explored the hypothesis that certain factors are critical to LLC implementation, feasibility, and satisfaction-namely, practice similarity (eg, same practice group or electronic health record [EHR]), geographic proximity, and existing relationship between clinicians. This article reports results for this secondary aim.

\section{Methods \\ QI Strategy}

The strategy used a combination of practice facilitation and LLCs. In the implementation phase (wave I), practices received assistance from an external facilitator, followed by a diffusion phase (wave II) in which members of wave I practices met with 2 other practices to focus on CKD guideline implementation. This approach involved translation and diffusion of strategies learned by early adopters (wave I clinician champions) to a second group of clinicians (wave II) during 6 monthly, 1-hour LLC meetings. Wave I clinicians were expected to be actively involved and to build on lessons learned in wave I. The LLCs received support from the practice facilitator $(\mathrm{PF})$, who coordinated meetings and helped practices provide monthly performance data. 


\section{Population and Setting}

Four regional PBRNs in Oklahoma (OKPRN), California (LANet), Wisconsin (WREN), and Minnesota (MAFPRN), and a research corporation (Westat), collaborated on the project; each PBRN had experience leading QI projects with PFs. Our goal was to support the formation of 8 LLCs within each PBRN, each with 3 practices (1 from wave I, 2 from wave II), with the potential reach of 96 practices.

\section{Process and Outcome Measurement}

The mixed-methods design structure was QUAN $\rightarrow$ QUAL, with sequential collection and analysis of quantitative and qualitative data. The function of the analysis was primarily expansion, whereby qualitative data addressed questions regarding project implementation raised by the quantitative data. ${ }^{26}$ LLC meeting and practice summaries were collected from the PFs in each PBRN, and data on guidelines worked on and fidelity were abstracted, including the number of meetings with (1) a representative from each practice, (2) a wave I clinician present, and (3) discussion of performance data. Because implementation was a function of the resources and constraints of each PBRN,,${ }^{27}$ coordinators were asked to write short descriptions of the feasibility of their implementation strategies, considering the context of the PBRN.

The quantitative fidelity data were used to identify 2 high- and 2 low-performing LLCs to collect data through follow-up interviews with participating clinicians in these practices. The interview guide addressed reactions to the LLCs and perceptions of changes in skills, knowledge, and clinical care. Participants were informed that responses would be kept private and reported in the aggregate, and consent to participate and audio-record the discussion was obtained in advance. Cleaned, formatted interview notes were checked against recordings for accuracy and imported into NVivo 9 (QSR International, Burlington, MA). Two coders identified potential codes of interest, based on the content areas and literature identifying drivers for successful LLCs, ${ }^{28,29}$ and developed a codebook with primary and secondary code names, definitions, and examples. The analysis process was initiated through regular analysis team meetings; disagreements in classification were discussed and resolved through consensus. The NVivo Matrix Coding query function was used to identify pat- terns and compare responses by PBRN and high/ low fidelity score. Queries also assessed whether themes were expressed across respondents to ensure that topics representative of multiple respondents' experiences were identified. Throughout the analysis process led by the first author (PDL), emergent themes were shared with the PBRN coordinators for feedback.

\section{Results}

With the assistance of the PBRNs, each wave I practice was grouped with 2 additional practices. Wave I practices were PBRN members interested in improving CKD care and willing to assist 2 other practices during the subsequent LLC intervention. Wave II practices were less likely to be a PBRN member and to have participated in a QI project, but they were otherwise similar to wave I practices and included private practices, hospital outpatient clinics, academic practices, and federally designated health centers or rural clinics. A total of 89 practices participated across both waves of the project (93\% of the target goal of 96 practices).

LLCs were implemented in 3 of the 4 PBRNs. Two collected complete meeting minutes, from which fidelity outcomes were extracted. Because the qualitative study was designed to stratify based on the assessment of high versus low fidelity, a subset of clinicians from these 2 PBRNs participated in the interviews. Table 1 summarizes LLC formation and the availability of data.

\section{Implementation Conduct, Fidelity, and Feasibility} LLC Conduct and Fidelity

Twenty LLCs were formed and met at least once. Although 8 LLCs were initially formed in LANet, none was able to meet as designed. OKPRN formed 8, WREN 7, and MAFPRN 5 LLCs. There were 121 monthly meetings held across 20 LLCs, with most holding the 6 required meetings (Table 2). A total of 210 unique primary care staff participated in at least 1 LLC meeting; WREN had the highest number of unique attendees. Slightly more than half $(54 \%)$ were clinicians, with the largest proportion of clinician participants $(68 \%)$ in OKPRN.

\section{Feasibility of the Implementation Strategy within Each PBRN}

Each PBRN coordinator provided a summary of the feasibility issues related to the implementation 
Table 1. Local Learning Collaborative (LLC) Formation and Availability of Data by Practice-based Research Network (PBRN)

\begin{tabular}{|c|c|c|c|c|c|}
\hline & \multirow[b]{2}{*}{ Total } & \multicolumn{4}{|c|}{ PBRN } \\
\hline & & LaNET & MAFPRN & OKPRN & WREN \\
\hline LLC formed* & 30 & 8 & 7 & 8 & 7 \\
\hline LLC met at least once & 20 & 0 & 5 & 8 & 6 \\
\hline Coordinator summaries & 4 & Yes & Yes & Yes & Yes \\
\hline Implementation outcomes assessed ${ }^{* *}$ & 3 & NA & Yes & Yes & Yes \\
\hline Intervention fidelity assessed ${ }^{* * *}$ & 2 & NA & NA & Yes & Yes \\
\hline Clinician perspectives & 2 & NA & NA & Yes & Yes \\
\hline
\end{tabular}

${ }^{*}$ LLC officially formed consisting of one representative from Wave I practice and a representative from each of the two Wave II practices.

${ }^{* *}$ LLC implementation occurred in three of four PBRNs.

***Two of three PBRNs collected complete meeting minutes from which fidelity outcomes were extracted. Clinicians from these two PBRNs participated in the interviews.

strategy for each PBRN. ${ }^{27}$ Statements are presented in their entirety (Table 3).

\section{LLC Fidelity Outcomes}

Data on LLC fidelity were extracted from minutes of 90 meetings held across 15 LLCs in 2 PBRNs (Table 4). At the majority of meetings, all practices were in attendance $(79 \%)$, the wave I clinician was present $(82 \%)$, and performance data were discussed (68\%).

\section{CKD Guidelines Targeted for Change}

During the intervention, some practices worked on as few as 3 guidelines, whereas others worked on as many as 6 (Table 5). Patient education (smoking cessation, counseling) was the guideline most frequently addressed, followed by adding CKD diagnosis to the problem list. Least frequently addressed was eGFR in patient charts. More OKPRN practices worked on adding appropriate medications and removing inappropriate medications (20 practices each), and fewer worked on eGFR in patient charts (8 practices). WREN practices tended to work on patient education most frequently (21 practices) and ordering of appropriate laboratory tests least frequently (7 practices).

\section{Clinician Experience (Qualitative Interview Data)}

Interviews were conducted with 21 of 24 clinicians in 8 LLCs (4 each in OKPRN and WREN). Using a modified grounded theory approach, the analysis was based on the first 2 levels of the 4-level evaluation framework developed by Kirkpatrick ${ }^{30}$ : (1) participants' reactions and (2) perceived change in knowledge, skills, and attitudes (Table 6). There were no findings related to LLC fidelity (comparing clinicians in LLCs with high- vs low-fidelity outcomes) or PBRN.

\section{Level 1: Participants' Reactions}

Three prominent themes include perceived impact of practice similarities and prior relationships, per-

Table 2. Implementation Outcomes Representing Intervention Conduct and Fidelity for Three LLCs. $(\mathrm{N}=20$ LLCs, 3 PBRNs)

\begin{tabular}{lcccc}
\hline & & \multicolumn{3}{c}{ PBRN } \\
\cline { 3 - 5 } Implementation outcome & Overall $(\mathrm{N}=20)$ & MAFPRN $(\mathrm{N}=5)^{*}$ & OKPRN $(\mathrm{N}=8)$ & WREN (N = 7) \\
\hline No. of LLC meetings & 121 & 31 & 48 & 42 \\
No. of LLCs with at least 6 meetings & 19 & 5 & 8 & 6 \\
No. of unique participants & 210 & 46 & 56 & 108 \\
No. of clinician participants & $112(53 \%)$ & $30(65 \%)$ & $38(68 \%)$ & $44(41 \%)$ \\
\hline
\end{tabular}

*Data on implementation outcomes was not available for two MAFPRN LLCs.

LLC, local learning collaborative; PBRN, practice-based research network. 
Table 3. Practice-based Research Network (PBRN) Coordinator Perspectives on Local Learning Collaborative (LLC) Feasibility

Coordinator statement

LaNet Despite initial success at launching several LLCs (had at least one meeting), they were not sustainable. Lack of a critical mass of participants including the Wave I expert resulted in discussions that were superficial and of minimal use, leading to a decrease in enthusiasm for future participation. The primary barrier was the demands of patient care, as clinicians had limited control of schedules, and clinics were often understaffed as all were federally qualified health centers. In response, LANet offered virtual LLCs on a standing bi-weekly schedule, inviting all LANet site champions (not just those originally in the LLC), and arranging for a primary care physician who was expert in CKD to provide perspective on clinical care and guideline implementation. While maintaining continuity across sessions through agendas and minutes was not feasible, LANet created a central database for information on practice goals and progress that was updated after each session. This information was used by the LANet meeting facilitator to stimulate discussion and encourage sharing of lessons learned. These modifications increased participation as well as participant satisfaction.

MAPRN

- MAFPRN formed seven LLCs (14 practices; six in urban settings, six in rural settings, and two in the suburbs). Many Minnesota clinicians are familiar with sharing and discussing performance data, based on several years of QI strategy discussions based on data available to health systems and the public. While LLCs were open to administrators and other staff, they were only in attendance at the three in-person LLCs, compared to the four using web-based meetings due to distance between practices. Web-based meetings, especially among clinicians unknown to each other, appeared to decrease the sense of urgency generated through in-person meetings, with attendance likely suffering. For these meetings the PF was on site with a Wave II practice, and scheduled additional meetings with staff to provide further information as needed. As large health systems dominate care delivery in Minnesota, a challenge to participation was lack of alignment between health system and PBRN research priorities. Overall, the LLC experience for MAFPRN clinicians was useful for improving CKD patient care.

OKPRN

- Half of OKPRN's eight LLCs were located in rural/small cities and others were mostly suburban. For three of the LLCs, the close proximity of the practices provided easy access to meetings, as practices were either in the same building or nearby. Three others shared the same health system infrastructure, with system modifications shared more easily among practices. Most meetings were scheduled in-person, however due to the press of patient care and other competing demands, only those in close proximity managed to attend and to bring other staff members with them. For others, conference calling was the usual default that allowed at least some participation. Meetings were usually scheduled at lunch time, planned and organized by the PF, and included lunch purchased by the Wave I clinician if the meeting was in person. The PF typically rotated attendance at one of the practices. Early sessions were recorded (with permission) to assess adherence to the meeting protocol and general direction of discussions. Two factors supported successful LLCs: prior personal relationships and geographic proximity. Most clinicians were known to each other and brought these relationships as an asset to LLC meetings. When prior relationships did not exist, LLCs were formed based on either common medical system or use of the same EHR. Practices close geographic proximity had the best attendance rates and staff participation, and in-person meetings compared to conference calls were easier to maintain over time.

WREN - Seven LLCs formed by WREN were a mix of rural and urban practices; not enough practices were recruited to form the eighth. Four were comprised of members of the same health care system or were co-located and able to meet in person. Geographic spread for the other three was too large for in-person meetings to be feasible, so they met using telephone conferencing. Although videoconference and Skype were attempted, they were not favored and were discontinued. Positive aspects of LLC participation included sharing of successes by Wave I clinicians with other LLC participants that resulted in implementation of these strategies within the Wave II practices. For example, a patient education document developed by an inner-city Wave I clinic was shared with all WREN practices and other participating PBRNs. Additional help was provided by the sharing of EHR enhancements and these continued after the project ended. Barriers to LLC success included both PF and leadership turnover. Two Wave I PFs and the original project coordinator were unavailable during Wave II, and this essential continuity was lost. In addition, some Wave I practices were more actively involved recruiting for the LLCs, which resulted in only seven successfully formed. For many practices this was their first WREN project, which meant that PBRN staff were facilitating the intervention at the same time they were building new relationships with staff. Thus, some of the intervention strategies were delayed and may not have had sufficient time to impact change in outcomes.

CKD, chronic kidney disease; QI, quality improvement; EHR, electronic health record.

ceptions of the feasibility of LLCs, and overall project satisfaction. Most observations reflected positive aspects of shared practice characteristics, such as the same EHR or practice group, as well as the impact of a prior relationship among clinicians. Practice dissimilarity was a barrier to LLC effectiveness: as 1 clinician explained, "in meetings we'd talk about what they could do, but 
Table 4. Fidelity Outcomes by Practice-based Research Network (PBRN) (N = 15 LLCs, 2 PBRNs)

\begin{tabular}{lccc}
\hline Fidelity outcomes & Total & OKPRN & WREN \\
\hline No. of LLC meetings & 90 & 48 & 42 \\
No. with all practices present (meetings) & $71(79 \%)$ & $37(77 \%)$ & $34(81 \%)$ \\
No. with Wave I clinician present (meetings) & $74(82 \%)$ & $46(96 \%)$ & $28(67 \%)$ \\
No. with performance data (meetings) & $61(68 \%)$ & $35(73 \%)$ & $26(62 \%)$ \\
\hline
\end{tabular}

LLC, local learning collaborative.

the clinic settings are different." Another participant commented that differences in patient volume may explain reluctance to implement suggestions for improvements in ordering lab work. One interviewee believed that implementing new strategies and altering processes was easier in smaller offices compared with larger ones, whereas others commented that larger practices had the advantage because of more resources and staff.

\section{Feasibility of LLCS}

The majority of comments referred to a lack of protected time to devote to the project, including meeting preparation. One clinician stated, "I was happy to make the commitment. It was difficult in practice to pull it off." In general, clinicians agreed that LLCs can be productive if groups are small, the topic is highly relevant, and the time commitment reasonable. Despite competing demands, participants were interested in attending, especially when meetings were held at their own sites at lunch time. Meeting frequency was considered adequate for both setting goals and assessing changes in performance data. The role played by the PFs in logistics and agenda-setting (and providing assis- tance between meetings, if needed), relevance of the CKD topic, a comfortable setting, and support from the entire practice team were identified as critical elements. Setting goals and deadlines at the first meeting was also considered helpful in achieving results.

The impact of sharing feedback data on motivation for improvement was a key finding. Even those less experienced with this strategy found the data helpful for comparing practices and monitoring their progress. Having clinical data available in advance was advised so clinicians had time to review the information and come prepared with questions. As 1 clinician stated, "The meetings were helpful to answer questions that did come up. In addition [they] conveyed level of accountability that helps keep you motivated."

\section{Satisfaction with the Project}

Most participants were highly satisfied, though typical demands of primary care practice had an impact: “. . . I would have gotten more out of it if I did not have so many other stressors." Another participant stated that reimbursement for time would also contribute to LLC success: "Anytime I am not seeing patients and am doing paperwork, [I am]

Table 5. CKD Guidelines Addressed by Practices in Two LLCs

\begin{tabular}{lccc}
\hline & & & PBRN \\
\cline { 3 - 4 } Guidelines & Total & OKPRN & WREN \\
\hline Number of practices, N & 45 & 24 & 21 \\
Guidelines worked on at least once, Range & 3 to 6 & 3 to 6 & $18(75 \%)$ \\
Patient education, N (\%) & $39(87 \%)$ & $16(67 \%)$ & $21(100 \%)$ \\
CKD diagnosis on problem list, N (\%) & $36(80 \%)$ & $20(83 \%)$ & $20(95 \%)$ \\
Addition of appropriate meds, N (\%) & $35(78 \%)$ & $20(83 \%)$ & $15(71 \%)$ \\
Removal of inappropriate meds, N (\%) & $31(69 \%)$ & $19(79 \%)$ & $11(52 \%)$ \\
Ordering of appropriate labs, N (\%) & $26(58 \%)$ & $8(33 \%)$ & $7(33 \%)$ \\
eGFR in patient charts, N (\%) & $25(56 \%)$ & $17(81 \%)$ \\
\hline
\end{tabular}

CKD, chronic kidney disease; eGFR, estimated glomerular filtration rate; LLC, local learning collaborative; PBRN, practice-based research network. 
Table 6. Themes and Examples from Clinician Interviews

Reaction

Sharing the same EHR

- . . "made a big difference. One of the biggest challenges is dealing with different EHRs. Knowing that we are all the same that way, because one little change can throw things off, even different sites that are the same clinic may have different SmartSets."

- . . "made it slightly easier in terms of talking about quality improvement or a workflow, especially trying to facilitate improvements with the EHR, everyone knows how it would work."

- "It's nice to share information, but to share monthly meetings with both clinics didn't make any sense because they were so different."

Belonging to the same practice group " "I think the fact that we were all from the same company, we all have the same difficulties with our patients. I think that helped us as far as being able to make suggestions to know what the other is going through and roadblocks we encounter."

- . . . "there's a certain level of comfort [among members of the same group], people are more open to share their opinions."

Key success factors

Sharing performance data

- . . "When you look at your own stats, you always think that they are going to be better than they are. And when you don't see it that way, you ask what you can do better."

- "We learned that there is a lot more we can do and have to be more attentive," and "... always good to see your data, and see how you stand. . . hopefully motivation to keep improving."

- "When you saw the numbers at the end and compared to the other clinics that was helpful because there is always a little competition I guess, which motivates staff at times."

Reaction

Team support

- "From my site, I was the only one who consistently took the time. ... you got to have the key players at the table, but I would think two or three so it feels like a team effort."

Satisfaction and sustainability

" "First experience, really enjoyed it."

- "It was really good. I'll see about signing up for another wave."

- "The project itself went well. The only thing now is keeping it going, the list updated, staying on top of CKD."

- "Didn't have the tools to keep the changes going long-term. Good for short-term. To affect patient care, needs to be implemented longer-term; don't have the tools to do that. Still don't today.'

Change in knowledge, skills, and attitudes

CKD knowledge and skills

- "I was shocked at how many patients fall into mild CKD, where their creatinine levels were normal, but their GFRs were low."

- "Just having it on the problem list with all my patients has really changed my practice."

- "We have so many diabetics on metformin, but I didn't make it a priority to check."

- "[It] helped keep staff and me more focused on what we're doing. . . . it helped us develop strategies to incorporate in daily work."

Priority for improving CKD care

- "It also made me much more confident that I knew what to look for and had some clue about what to do with what I found."

- "... definitely made a difference in terms of my priority. I wasn't really clear about the management and treatment and identification of CKD. And this study really put things in focus when we discussed the numbers [feedback reports]."

CKD, chronic kidney disease; EHR, electronic health record; GFR, glomerular filtration rate.

going in the hole. If I have to do that and was not reimbursed, it is not practical." Two clinicians noted that an incentive, such as lunch at the time of meeting, "just brings members right on in" and would "improve the morale."

\section{Level 2: Changes in Knowledge, Skills, and Attitudes}

Increasing knowledge of CKD was cited frequently as the most significant outcome, particularly CKD screening and the effect of nonsteroidal anti-inflammatory drugs. Meetings had a positive impact on perceived ability to care for patients with CKD. Several clinicians reported improvements in patient education skills and protocols to identify and contact at-risk patients. For those with protocols or systems already in place, participating in meetings provided additional reinforcement and feedback. The LLC meetings were found to be less effective 
for clinicians who were already knowledgeable about CKD or perceived that they were already meeting expectations for effective CKD care.

\section{Priority for Improving CKD Care}

Participation also increased awareness of CKD care by providing a better understanding of the condition and increased confidence to identify and treat it. Some interviewees appreciated reinforcement on the importance of CKD, and others stated that this new information supplemented medical school training.

\section{Discussion}

This mixed-methods study provides different perspectives on a peer-to-peer intervention approach, facilitated by PBRNs, intended to affect clinician performance. Quantitative results show that the LLCs were not implemented as planned in every PBRN and that practice participation was challenging. Qualitative data suggest that clinicians increased priority for CKD care, improved knowledge and skills, were satisfied with the project, and attempted to improve care. Implementation challenges were encountered and concerns about sustainability expressed through a variety of sources, including LLC meeting summaries (from the PF perspective), coordinator descriptions, and discussions with participating clinicians. The wealth of data generated through this multi-PBRN project provides concrete reflections on the challenges associated with achieving CKD-related QI goals, as well as practical advice for the design and implementation of similar collaborative QI interventions. In particular, clinicians confirmed that sharing performance data, a key element of the LLC design, was critical to the effectiveness of the intervention and confirmed the importance of social relations and trust in establishing a productive learning environment. ${ }^{31,32}$ Not surprisingly, travel was a barrier to LLC attendance, even when meetings were scheduled at lunch time, and meetings held either by video or telephone were perceived as less effective unless groups were known to each other.

QI goals targeted by practices were often PBRN-specific, given regional differences in practice oversight and particular practice standards and influences, as described from the perspective of the PBRN coordinators. The data show that patient education was most frequently addressed, perhaps because clinicians in these particular PBRNs (OKPRN and WREN) did not have educational materials and appreciated access to these resources, and that the goal was relatively easy to achieve. Having a clear diagnosis in the problem list also emerged as a frequent goal, as specific care protocols were triggered by a CKD diagnosis and associated with having an eGFR value in the chart. For some, this was seamless when lab values were posted in the EHR by the cooperating laboratory; others found the required levels of bureaucracy made this goal unachievable under existing constraints. Based on our prior experience, achievements in the targeted QI goals were ones that were likely to be sustained.

\section{Conclusions}

PBRNs are unique environments in which to foster opportunities to improve the quality of primary care and to facilitate the sharing of evidence-based and practical solutions to common problems. While PBRNs can successfully leverage resources to disseminate treatment guidelines, and LLCs are well accepted by clinical staff, the formation of LLCs may not be feasible or effective in all contexts, even with logistic support. Data collected in this multi-PBRN project indicate that sharing performance data created motivation to improve patient care, attendees learned valuable lessons from each other, and clinicians were actively involved in attempts to improve the implementation of CKD guidelines.

In this article we have outlined a mixed-methods approach to assessing clearly defined intervention fidelity criteria. A key finding is confirmation from the perspectives of coordinators and clinicians that designing LLCs with certain factors in mind motivates a learning collaborative and contributes to feasibility and participant satisfaction. LLCs were perceived as more successful when there were prior relationships and when there was either more geographic proximity between practices in a single LLC and/or a health system infrastructure common to all. The coordinators and PFs observed that the likelihood of disseminating a best practice from 1 setting to another was enhanced under these conditions.

The authors appreciate the contributions of PBRN project coordinators Melody Bockenfeld (WREN), Carol Lange (MAFPRN), and Lyndee Knox (LANet), who provided their perspective on LLC implementation. The authors also greatly appreciate the practice facilitators, who provided the meeting minutes on which these analyses are based, and the practice staff who par- 
ticipated in the interviews. At Westat, Dr. Jennifer Huang led the qualitative task and conducted interviews with Vibha Vij, and Jeffrey Elmer served as coordinator.

\section{References}

1. Kidney disease statistics for the United States. NIH publication no. 12-3895. Bethesda, MD: National Kidney and Urologic Diseases Information Clearinghouse; 2012. Available from: http://kidney. niddk.nih.gov/kudiseases/pubs/kustats/KU_Diseases_ Stats_508.pdf. Accessed July 21, 2016.

2. Keith DS, Nichols GA, Guillion CM, et al. Longitudinal follow-up and outcomes among a population with chronic kidney disease in a large managed care organization. Arch Intern Med 2004; 164:659-63.

3. Tuot DS, Grubbs V. Chronic kidney disease care in the U.S. safety net. Adv Chronic Kidney Dis 2015; 22:66-73.

4. National Kidney Foundation. K/DOQI clinical practice guidelines for chronic kidney disease: evaluation, classification, and stratification. Am J Kidney Dis 2002;39(2 Suppl 1):S1-266.

5. Jafar TH, Stark PC, Schmid CH, et al. The effect of angiotensin-converting enzyme inhibitors on progression of advanced polycystic kidney disease. Kidney Int 2005;67:265-71.

6. Gooch K, Culleton BF, Manns BJ, et al. NSAID use and progression of chronic kidney disease. Am J Med 2007;120:280.e1-7.

7. Gouva C, Nikolopoulos P, Ioannidis JP, et al. Treating anemia early in renal failure patients slows the decline of renal function: a randomized controlled trial. Kidney Int 2004;66:753-60.

8. Shepherd J, Kastelein JJ, Bittner V, et al. Intensive lipid lowering with atorvastatin in patients with coronary heart disease and chronic kidney disease: the TNT (Treating to New Targets) study. J Am Coll Cardiol 2008;51:1448-54.

9. Greer R, Boulware LE. Reducing CKD risks among vulnerable populations in primary care. Adv Chronic Kidney Dis 2015;22:74-80.

10. Mendu M, Schneider LI, Aizer AA, et al. Implementation of a CKD checklist for primary care providers. Clin J Am Soc Nephrol 2014;9:1526-35.

11. Liu C, Chen H, Liu C, et al. Combined application of eGFR and albuminuria for the precise diagnosis of stage 2 and 3a CKD in the elderly. J Nephrol 2014; 27:289-97.

12. Abdel-Kader K, Greer RC, Boulware LE, Unruh ML. Primary care physicians' familiarity, beliefs, and perceived barriers to practice guidelines in nondiabetic CKD: a survey study. BMC Nephrol 2014; 15:64.

13. Schonlau M, Mangione-Smith R, Chan KS, et al. Evaluation of a quality improvement collaborative in asthma care: does it improve processes and outcome of care? Ann Fam Med 2005;3:200-8.
14. Hulscher ME, Wensing $M$, van Der Weijden T, et al. Interventions to implement prevention in primary care. Cochrane Database Syst Rev 2001;1: CD000362.

15. Mangione-Smith R, Schonlau M, Chan KS, et al. Measuring the effectiveness of a collaborative for quality improvement in pediatric asthma care: does implementing the chronic care model improve processes and outcomes of care? Ambul Pediatr 2005;5:75-82.

16. Grimshaw JM, Thomas RE, MacLennan G, et al. Effectiveness and efficiency of guideline dissemination and implementation strategies. Health Technol Assess 2004;8:iii-iv, 1-72.

17. Curran GM, Bauer M, Mittman B, et al. Effectiveness-implementation hybrid designs: combining elements of clinical effectiveness and implementation research to enhance public health impact. Med Care 2012;50:217-26.

18. Accreditation Council for Continuing Medical Education (ACCME) annual report data 2012. Available from: hhttp://www.accme.org/news-publications/publications/ annual-report-data/accme-annual-report-2012. Accessed July 21, 2016.

19. Translating research into practice (TRIP)-II. Washington, DC: Agency for Healthcare Research and Quality; 2001.

20. McMullen CK, Schneider J, Firemark A, et al. Cultivating engaged leadership through a learning collaborative: lessons from primary care renewal in Oregon safety net clinics. Ann Fam Med 2013;11(Suppl 1):S34-40.

21. Lineker SC, Bell MJ, Boyle J, et al. Implementing arthritis clinical practice guidelines in primary care. Med Teach 2009;31:230-7.

22. Ayers LR, Beyea SC, Godfrey MM, et al. Quality improvement learning collaboratives. Qual Manag Health Care 2005;14:234-47.

23. Roosa M, Scripa JS, Zastowny TR, Ford JH 2nd. Using a NIATx based local learning collaborative for performance improvement. Eval Program Plann 2011;34:390-8.

24. Aspy CB, Mold JW, Thompson DM, et al. Integrating screening and interventions for unhealthy behaviors into primary care practices. Am J Prev Med 2008;35:S373-80.

25. Mold JW, Aspy CB, Smith P, et al. Leveraging PBRNs to accelerate implementation and diffusion of chronic kidney disease guidelines in primary care practices. Implement Sci 2014;9:169-79.

26. Cresswell JW, Plano Clark VL. Designing and conducting mixed method research. Thousand Oaks, CA: Sage; 2007.

27. Aspy CA, Lipman PD, Bockenfeld M. Lessons learned in multi-network quality improvement research using local learning collaboratives to disseminate practice guidelines: case studies from a CKD quality improvement project in primary care. Presented at a workshop at the Annual Meeting of the North American Primary 
Care Research Group (NAPCRG), November 2013, Ottawa, Ontario, Canada.

28. Joly BM, Booth M, Shaler G, Conway A. Quality improvement learning collaboratives in public health: findings from a multisite case study. J Public Health Manag Pract 2012;18:87-94.

29. Joly BM, Booth M, Shaler G, Conway A. Developing and managing quality improvement learning collaboratives: lessons from the MLC states. Washington, DC: National Network of Public Health Institutes; 2010.
30. Kirkpatrick DL. Evaluating training programs: the four levels. San Francisco: Berrett-Koehler Publishers Inc.; 1994.

31. Miller WL, Crabtree BF, Nutting PA, et al. Primary care practice development: a relationshipcentered approach. Ann Fam Med 2010;8(Suppl 1):S68-79.

32. Lanham HJ, Palmer RF, Leykum LK, et al. Trust and reflection in primary care practice redesign. Health Serv Res 2016;51:1489-514. 